\title{
Factores asociados al autorreporte del bienestar mental o emocional del hombre en Chile
}

\author{
Carolina Vidal ${ }^{1}$ y Carol Toro-Huerta ${ }^{1}$
}

Forma de citar Vidal C, Toro-Huerta C. Factores asociados al autorreporte del bienestar mental o emocional del hombre en Chile. Rev Panam Salud Publica. 2018;42:e181. https://doi.org/10.26633/RPSP.2018.181

RESUMEN Objetivo. Determinar factores asociados al bienestar mental o emocional del hombre en Chile.

Métodos. Estudio analítico transversal en hombres de 15 o más años en Chile. Se utilizaron datos de la Encuesta Nacional de Calidad de Vida y Salud 2015-2016 (ENCAVI). Las variables de interés correspondieron a la percepción del bienestar emocional o mental y el autorreporte de depresión o ansiedad. Se estimó la prevalencia de cada una de ellas. Se evaluó la asociación de las variables de interés con factores sociodemográficos, hábitos y salud mediante regresión logística.

Resultados. El 22,6\% (intervalo de confianza de 95\% [IC95\%]: 20,3\%-25,0\%) reportó una percepción de bienestar mental o emocional menos que buena, y 4,9\% (IC95\%: 3,8\%-6,4\%) declaró diagnóstico médico de depresión. La menor percepción de bienestar emocional estuvo asociada al reporte de una satisfacción sexual menos que buena, con una razón de momios (OR, por sus siglas en inglés) de 4,42 (IC95\%: 3,40-5,76) seguida de calidad de sueño y apoyo social menor a lo óptimo. Se hallaron resultados similares en quienes autorreportaron depresión o ansiedad; aqui, la mayor probabilidad de este autorreporte estuvo asociada a una calidad de sueño menor que buena, presencia de al menos una enfermedad crónica y menor satisfacción sexual.

Conclusión. El principal factor asociado al autorreporte de bienestar mental o emocional fue la insatisfacción con su vida sexual. Dicho resultado concuerda con que la identidad masculina se vincula con los desempeños sexuales. Es necesario avanzar en la investigación de la salud de los hombres y proporcionar orientación para iniciativas de políticas de salud.

Palabras clave Hombres; masculinidad; salud del hombre; Chile.

La incorporación de políticas de salud con perspectiva de género es un

\footnotetext{
1 Escuela de Salud Pública, Universidad Mayor, Santiago, Chile. Enviar correspondencia a Carol

Toro-Huerta, caroltoroh@gmail.com
}

importante desafío para los sistemas de salud. La evidencia señala que hombres y mujeres presentan diferentes necesidades y desenlaces en salud (1). Sin embargo, para abordar estos procesos resulta insuficiente solo considerar indicadores relacionados a morbilidad, mortalidad y causas de muerte. Por ello, y en consideración al aumento de la expectativa de vida de la población, se hace necesario obtener información acerca de la calidad de vida de las personas en sus dominios físico, mental y social (2). 
El concepto de calidad de vida ha sido difícil de definir, se reconoce que es un término multidisciplinario y que su evaluación podría enfocarse desde una dimensión objetiva, relacionada al bienestar material o desde una perspectiva subjetiva que hace referencia a la percepción individual que tienen los individuos en su calidad de vida. Incluido en esta última, se encuentra el bienestar emocional, satisfacción con la vida o felicidad, términos que han sido utilizados como sinónimos en la literatura (3).

El bienestar emocional se encuentra asociado a las experiencias del individuo (4) y reflejan una combinación de sensaciones positivas y satisfacción general con la vida $(5,6)$. Asimismo, el bienestar emocional implicaría un funcionamiento sicológico óptimo, por lo que se lo considera una dimensión de la salud mental que actúa de forma independiente de la enfermedad mental, aunque tienen una relación muy estrecha (7).

Muchas de las diferencias de salud entre hombres y mujeres se remontan a la socialización masculina, que tiene un impacto en las elecciones de los estilos de vida (8). Se ha identificado que las conductas, hábitos y estilos de vida afectan el bienestar de forma diferenciada a hombres y mujeres, lo que se debe en parte a los aspectos biológicos particulares para cada sexo, pero también al rol asociado a las construcciones de género (9). Esto último explicaría, por ejemplo, que los hombres sean proclives a adoptar creencias y conductas de riesgo para la salud (10). En promedio, los hombres fuman tres cigarrillos más por día y presentan una mayor dependencia al tabaco que las mujeres (11). Además, presentan un mayor consumo de alcohol, el cual se asocia a otros comportamientos poco saludables, como la actividad sexual de alto riesgo, riesgo de enfermedades de transmisión sexual y pensamientos suicidas (12). El consumo de alcohol presenta una alta comorbilidad con el uso de drogas, trastorno depresivos, ansiedad, trastorno del estado de ánimo y conducta $(13,14)$. Una revisión de la literatura respecto de la salud de los hombres indica que los factores biológicos presentan un menor peso en la predicción de la morbilidad y mortalidad, en relación con los factores sociales y psicológicos (15). Algunos factores que se han reportado como asociados a depresión corresponden a clases sociales menos favorecidas, menor nivel educacional, ser soltero, separado, viudo o divorciado (16-18).
En este marco, las características que se atribuyen a lo masculino y femenino posicionan al hombre en una condición hegemónica dentro de la sociedad (19). Los hombres son menos expresivos que las mujeres con sus emociones en general; sin embargo, se observa una exacerbación en las expresiones relacionadas con la ira. Esto tendría efectos en el bienestar y en la salud física de los hombres y se manifiesta en un mayor riesgo enfermedades cardiovasculares, alteraciones del sueño y manifestación de estrés $(20,21)$. También los lugares de trabajo son sitios clave para la construcción de género (22). Los hombres se encuentran expuestos a resultados adversos en salud, con altos niveles de estrés y la búsqueda de apoyo externo poco frecuente (23).

El propósito de este estudio es determinar los factores asociados al autorreporte de bienestar emocional en hombres chilenos de 15 y más años según los datos de la Encuesta de Calidad de Vida y Salud (ENCAVI) 2015-2016.

\section{MATERIALES Y MÉTODOS}

Se trata de un estudio analítico transversal; la población de estudio corresponde a hombres de 15 o más años que residen en viviendas particulares ocupadas localizadas en zonas urbanas y rurales de las quince regiones de Chile. Los datos fueron obtenidos de la ENCAVI 2015-2016, realizada por el Ministerio de Salud de Chile (24). Esta encuesta es representativa a nivel nacional, y utilizó un muestreo probabilístico, estratificado geográficamente y multietápico, con un tamaño muestral alcanzado de 7041 personas de 15 o más años, de los cuales 2624 registros corresponden a hombres con un rango de edad entre 15 y 93 años.

Se utilizaron dos variables de resultado: autorreporte de bienestar mental o emocional y autorreporte del diagnóstico de depresión o ansiedad. Para la primera se utilizó una pregunta de la encuesta, en la que se solicita al encuestado calificar con una nota del 1 al 7 respecto de qué tan bien se siente con su bienestar mental o emocional. Dicha variable se dicotomizó en las categorías muy bien o bien (nota 6 y 7) por una parte y, por otra, el resto de las notas (1 a 5). La segunda variable se refería a si alguna vez en la vida había sido diagnosticado con depresión o ansiedad por parte de un médico.
Los factores posiblemente asociados e incorporados al análisis fueron variables sociodemográficas, como el nivel educacional alcanzado medido en número de años de escolaridad, la situación laboral y de pareja, considerándose con pareja aquellos que tuviesen una relación de convivencia formal (casados, convivientes y conviviente civil) o informal (conviviente de hecho) y sin pareja aquellos que no pertenecían a ninguno de estos grupos. La edad se agrupó en tres categorías correspondientes a menores de 30 años, entre 30 a 59 años y 60 o más años; estas categorías se definieron en función de las diferentes etapas del ciclo vital y para permitir la comparabilidad con otras investigaciones. Respecto de los factores de los estilos de vida se incluyeron las variables consumo de tabaco durante el último mes y el consumo de alcohol medido a través del test abreviado de identificación de trastornos debido al consumo de alcohol (AUDIT, por sus siglas en inglés). Este test identifica a la persona que presenta un consumo de riesgo, consumo perjudicial o dependencia de alcohol (25). El estado de salud declarado se incorporó mediante el reporte de diagnóstico médico de al menos una de estas enfermedades o condiciones: diabetes, hipertensión arterial o enfermedad pulmonar obstructiva crónica. También se consideró la valoración que el encuestado realizó de la calidad del sueño que había tenido durante el último año, dicotomizada en las categorías buena (muy buena y buena) y menos que buena (muy mala, mala y regular). El apoyo social del encuestado se determinó a través de la pregunta: Cuando tiene problemas ¿tiene usted alguna persona en la que pueda confiar, pedir ayuda o consejos? Se consideró como presencia de apoyo social aquellos cuya frecuencia era de siempre y casi siempre y con bajo apoyo social quienes respondieron rara vez, nunca o no necesita. Se incluyó la satisfacción con la vida sexual a partir de la pregunta ¿Cuán satisfecho está con su vida sexual? Se consideró satisfecho aquellos que respondieron satisfecho y muy satisfecho e insatisfecho las categorías con una menor satisfacción. Por último, se incluyó la presencia de discriminación cuando se había sentido discriminado por al menos uno de los 15 motivos consultados.

Los análisis se realizaron aplicando los factores de expansión asociados al diseño muestral de la encuesta. Para describir a la población en estudio se utilizaron 
tablas de frecuencia con proporciones e intervalos de confianza del $95 \%$. Se estimó la prevalencia tanto para el autorreporte del bienestar mental o emocional como para el autorreporte del diagnóstico de depresión o ansiedad. Se utilizaron gráficos para evaluar las diferencias observadas en la percepción de discriminación de los hombres según su bienestar mental o emocional. Para determinar los factores asociados a ambos autorreportes, se utilizó un modelo de regresión logística con los factores antes descritos. Se calcularon los odds ratio (OR) y sus intervalos de confianza de 95\% (IC95\%). Además, se analizó el subgrupo de hombres en situación laboral de ocupados en el que se incluyeron variables de condiciones de trabajo en relación con la exposición de riesgos físicos y psicológicos.

Para este estudio se utilizó una base de datos de uso público, obtenido del Ministerio de Salud de Chile, por lo que los datos fueron confidenciales y anónimos según lo dispuesto en la Ley $\mathrm{N}^{\circ} 17374$ artículo 29 (26).

\section{RESULTADOS}

Un 30,9\% de los hombres presentó una edad inferior a los 30 años. Un $66 \%$ se presentó situación laboral de ocupado. Respecto a los estilos de vida se observó que $34,7 \%$ era fumador actual y $11,7 \%$ presentó conducta de riesgo asociada al alcohol. En cuanto a la discriminación, 23,1\% de los participantes declaró sentirse discriminado (cuadro 1).
La estimación de las variables de resultado se presenta en el cuadro 1, en el que se observa que 22,6\% (IC95\%: 20,3\%$25,0 \%$ ) de los hombres declaró un bienestar mental o emocional menos que bueno; mientras que 4,9\% (IC95\%: 3,8\%$6,4 \%$ ) declaró el diagnóstico médico de depresión o ansiedad (figura 1). En la figura 2 se muestran las razones por las cuales se ha sentido discriminados, la primera razón corresponde al nivel socioeconómico, con $12,1 \%$ en aquellos hombres que presentan un bienestar mental o emocional menos que bueno. Luego se describen las razones de edad y el lugar de residencia.
En el cuadro 1 se muestra la evaluación de la asociación entre los factores y las variables de resultado (autorreporte de bienestar mental o emocional y autorreporte de depresión o ansiedad). Los factores que resultaron asociados con mayor probabilidad de presentar bienestar emocional menos que bueno fueron, en orden decreciente: insatisfacción con su vida sexual (OR: 4,42; IC95\%: 3,40$5,76)$, la valoración en la calidad del sueño menos que buena (OR: 3,10; IC95\%: $2,41-3,98)$ y la percepción de bajo apoyo social (OR: 1,68; IC95\%: 1,27-2,22). Otros factores asociados, pero en menor magnitud corresponden a la presencia de al

\section{FIGURA 1. Distribución de frecuencia de bienestar mental o emocional y diagnóstico de depresión o ansiedad}

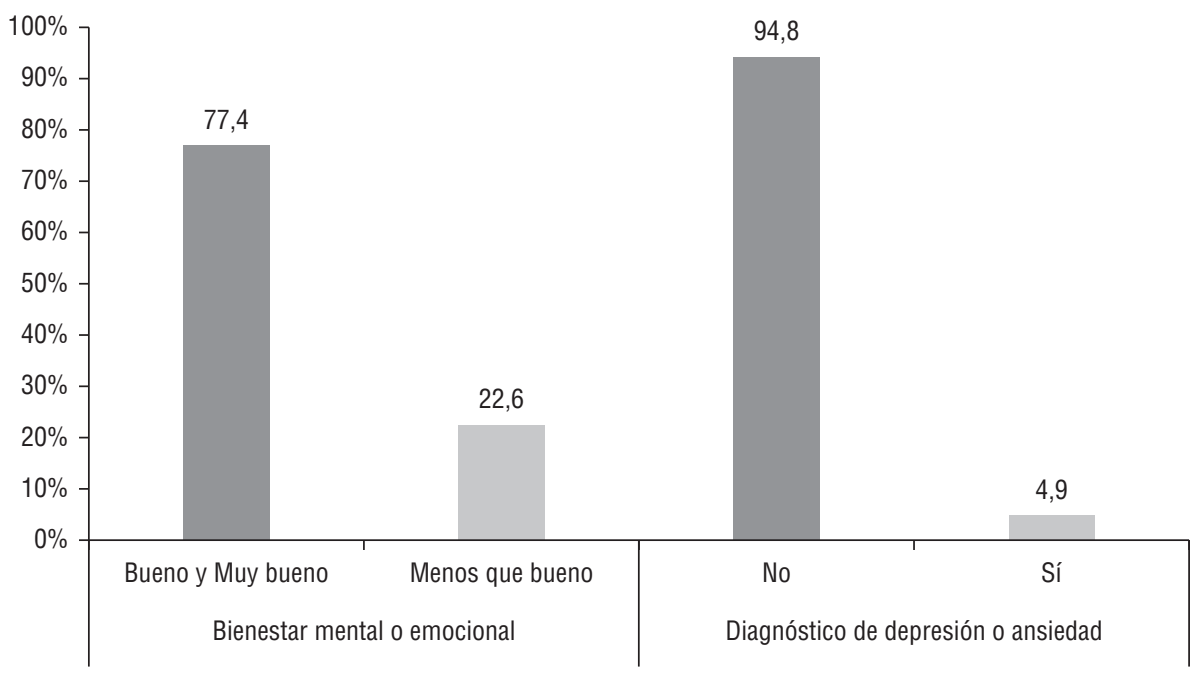

Elaboración propia a partir de los datos publicados ENCAVI 2015-2016.

FIGURA 2. Distribución de las razones de discriminación según el bienestar mental

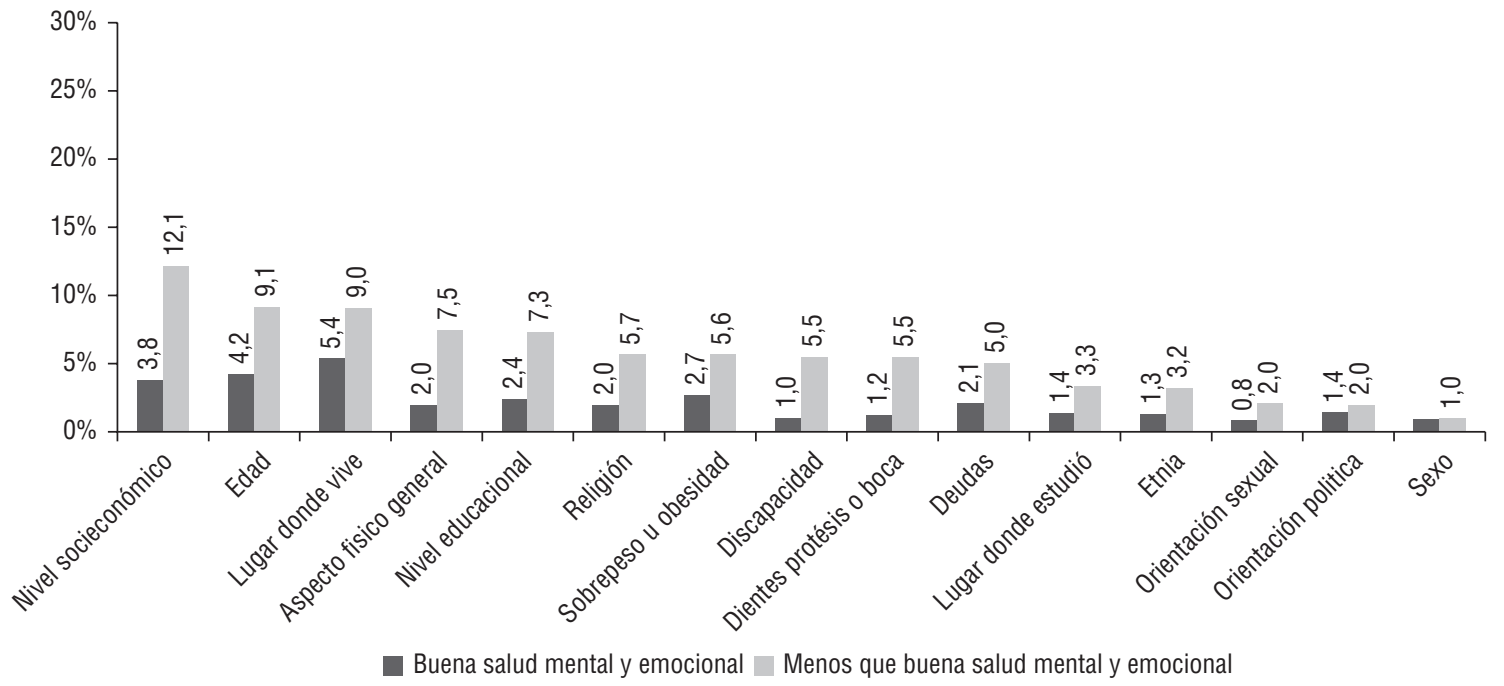

Elaboración propia a partir de los datos publicados ENCAVI 2015-2016. 
CUADRO 1. Factores asociados al autorreporte del bienestar mental o emocional y del autorreporte del diagnóstico médico de depresión o ansiedad del hombre, Chile

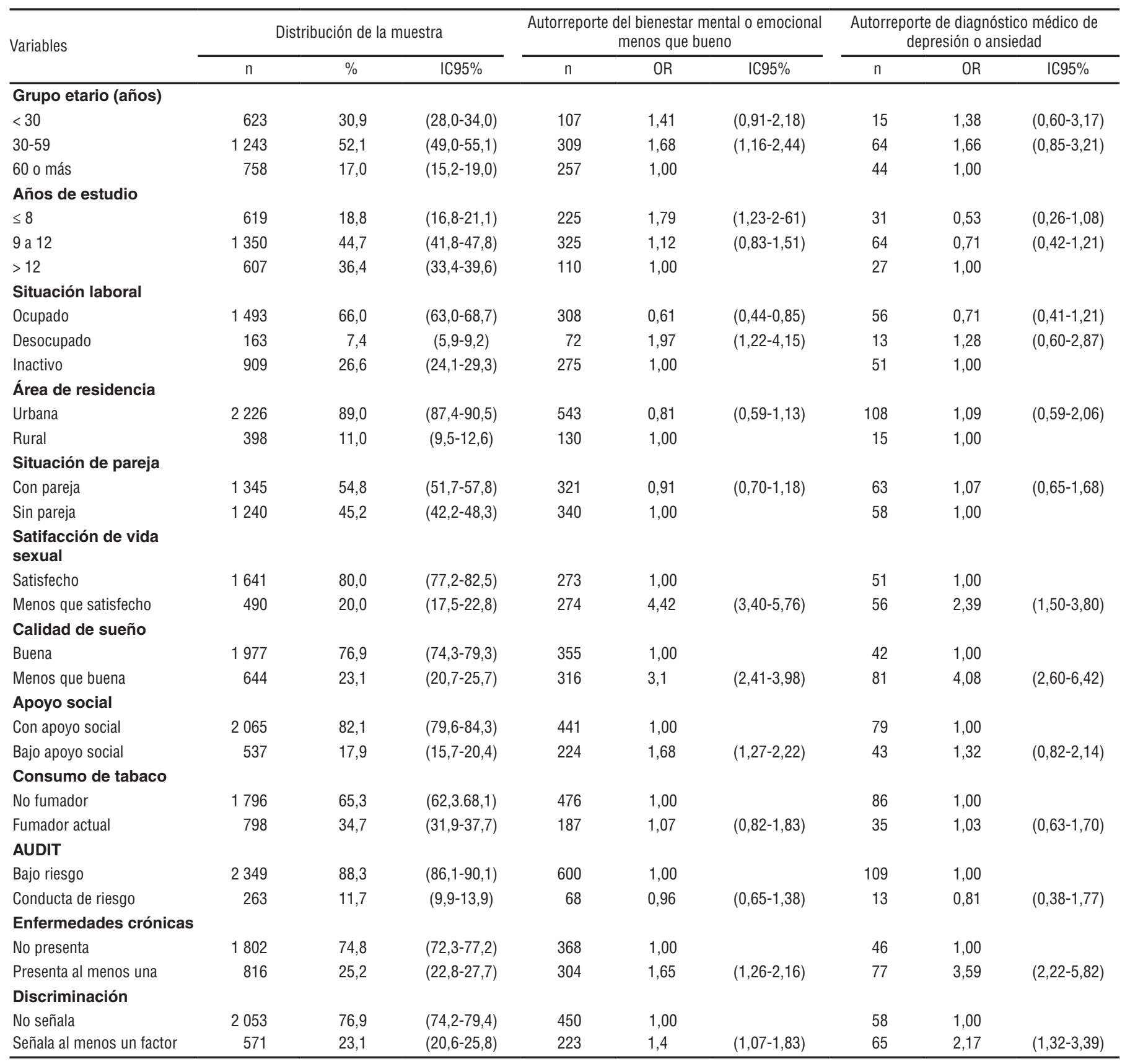

IC95\%, intervalo de confianza de 95\%; OR, odds ratio.

Elaboración propia a partir de los datos publicados en ENCAVI 2015-2016.

menos una enfermedad crónica, edad, nivel educacional y una situación laboral de desocupado.

Por otra parte, la probabilidad de autorreporte de diagnóstico médico de depresión o ansiedad es mayor en aquellos hombres que declaran una menor calidad del sueño (OR: 4,08; IC95\%: 2,60-6,42), presencia de al menos una enfermedad crónica (OR: 3,59;
IC95\%: 2,22-5,82) y menor satisfacción con su vida sexual (OR: 2,39; IC95\%: 1,50-3,80).

En el cuadro 2 se muestran los resultados obtenidos del análisis realizado en el subgrupo de hombres con situación laboral de ocupado; se observa que $70,9 \%$ se encuentra en el rango de edades entre 30 y 59 años. Un $41,6 \%$ ha consumido cigarrillos durante el último mes, 51,3\% calificó su situación económica menos que buena. En el análisis de este subgrupo se hallaron resultados similares a los obtenidos en los que se consideró a la totalidad de los hombres. En primer lugar, se encuentra la autocalificación de una menor satisfacción con su vida sexual (OR: 3,23; IC95\%: 2,284,59 ) y percepción económica (OR: 3,07; IC95\%: 2,19-4,30). 
CUADRO 2. Factores asociados al autorreporte del bienestar mental o emocional del hombre con situación laboral de ocupado, Chile

\begin{tabular}{|c|c|c|c|c|c|c|}
\hline \multirow{2}{*}{ Variables } & \multicolumn{3}{|c|}{ Distribución de la muestra de hombres con alguna ocupación } & \multicolumn{3}{|c|}{ Bienestar mental o emocional menos que bueno } \\
\hline & $\mathrm{n}$ & $\%$ & IC95\% & $\mathrm{n}$ & OR & IC95\% \\
\hline \multicolumn{7}{|l|}{ Grupo de etario (años) } \\
\hline$<30$ & 240 & 20,1 & $(17,0-23,6)$ & 49 & 1,71 & $(0,91-3,24)$ \\
\hline 60 o más & 190 & 9 & $(7,2-11,0)$ & 39 & 1,00 & \\
\hline \multicolumn{7}{|l|}{ Años de estudio } \\
\hline$>12$ & 421 & 40,5 & $(36,6-44,5)$ & 64 & 1,00 & \\
\hline \multicolumn{7}{|l|}{ Área de residencia } \\
\hline Urbana & 1260 & 87,7 & $(85,4-89,7)$ & 255 & 0,84 & $(0,55-1,29)$ \\
\hline Rural & 233 & 12,3 & $(10,3-14,6)$ & 53 & 1,00 & \\
\hline \multicolumn{7}{|c|}{ Satifacción de vida sexual } \\
\hline Satisfecho & 1122 & 83,1 & $(79,8-86)$ & 163 & 1,00 & \\
\hline Menos que buena & 329 & 21,7 & $(18,8-25)$ & 136 & 2,60 & $(1,87-3,63)$ \\
\hline \multicolumn{7}{|l|}{ Apoyo social } \\
\hline Con apoyo social & 1193 & 82,9 & $(79,8-85,5)$ & 206 & 1,00 & \\
\hline Bajo apoyo social & 291 & 17,1 & $(14,5-20,2)$ & 101 & 1,65 & $(1,15-2,37)$ \\
\hline \multicolumn{7}{|l|}{ Consumo de tabaco } \\
\hline No fumador & 903 & 59 & $(55,1-62,7)$ & 184 & 1,00 & \\
\hline Fumador actual & 573 & 41 & $(37,3-44,9)$ & 116 & 1,11 & $(0,80-1,52)$ \\
\hline \multicolumn{7}{|l|}{ AUDIT } \\
\hline Bajo riesgo & 1293 & 86,8 & $(84,1-89,1)$ & 263 & 1,00 & \\
\hline Conducta de riesgo & 192 & 13,2 & $(10,9-15,9)$ & 42 & 0,785 & $(0,49-1,26)$ \\
\hline \multicolumn{7}{|l|}{ Enfermedades crónicas } \\
\hline Menos que buena & 732 & 51,3 & $(47,5-55,1)$ & 233 & 3,07 & $(2,19-4,30)$ \\
\hline \multicolumn{7}{|l|}{ Riesgo psicológico } \\
\hline No expuesto & 510 & 35,9 & $(32,2-39,7)$ & 92 & 1,00 & \\
\hline Expuesto & 983 & 64,1 & $(60,3-67,8)$ & 216 & 0,762 & $(0,48-1,22)$ \\
\hline \multicolumn{7}{|l|}{ Riesgo físico } \\
\hline No expuesto & 796 & 56,6 & $(52,8-60,3)$ & 137 & 1,00 & \\
\hline Expuesto & 697 & 43,4 & $(39,7-47,2)$ & 171 & 1,38 & $(0,89-2,14)$ \\
\hline
\end{tabular}

IC95\%, intervalo de confianza de 95\%; OR, odds ratio.

Elaboración propia a partir de los datos publicados en ENCAVI 2015-2016.

\section{DISCUSIÓN}

La Organización Mundial de la Salud (OMS) reconoce la necesidad de prestar atención a las necesidades de salud específicas de los hombres. Para ello, es necesario comprender cómo el género, socialización e ideología masculina afectan la salud de los hombres (8).
Un $22,6 \%$ de los encuestados declaran un bienestar mental o emocional menos que bueno, y su principal factor asociado fue la insatisfacción con su vida sexual. Dicho resultado concuerda al pensar que la construcción de identidad masculina tiene una vinculación estrecha con los desempeños sexuales y la sexualidad de los hombres (27). De modo similar, un estudio realizado en Chile muestra que aquellos hombres que adhieren al estereotipo masculino experimentan niveles más bajos de bienestar psicológico (28).

La encuesta internacional de masculinidades y equidad de género (IMAGES, por sus siglas en inglés) (29), también aporta información interesante en este sentido y señala que quienes obtuvieron puntajes más bajo en la escala GEM (interpretado como actitudes de género 
menos equitativas por parte de los hombres) también reportaron sentirse deprimidos, con pérdida de deseo sexual o pensamientos suicidas. Por otra parte, quienes obtuvieron puntajes más altos, interpretado como actitudes de género más equitativas, señalaron tener una mejor vida sexual (29). Este ámbito se vuelve aun más relevante en la salud de los hombres si se toma en cuenta que son ellos quienes presentan con mayor frecuencia de conductas de riesgo para la salud sexual (30).

En las últimas décadas, ha surgido evidencia respecto de las diferencias en la morbilidad psiquiátrica y patrones de conducta desarrollados por hombres y mujeres $(20,31)$. Por otra parte, los estudios señalan que la depresión entre los hombres podría estar subdiagnosticada y sin tratamiento debido a las diferencias de género en los síntomas y el afrontamiento (31). El presente estudio estimó que el autorreporte del diagnóstico médico de depresión o ansiedad corresponde a $4,9 \%$. Similar proporción arroja el reciente Estudio Longitudinal Social de Chile (ELSOC), que reporta una prevalencia de síntomas de depresión más graves de $5 \%$ para hombres y de $9 \%$ para mujeres (32). Sin embargo, es importante destacar que, a pesar de que las tasas de depresión masculina son más bajas en comparación a las mujeres, esto no refleja necesariamente una mejor salud mental, ya que los hombres exhiben tasas bastante más altas de uso de sustancias y violencia física (31). La calidad del sueño tuvo una fuerte asociación con el autorreporte del diagnóstico médico de depresión o ansiedad. La calidad del sueño ha sido relacionada con depresión, enfermedad cardiovascular y deficiencias moderadas a graves de los componentes físicos y mentales del SF-36 (33); sin embargo, podría existir bidireccionalidad.

Los lugares de trabajo son sitios clave donde las identidades de género se construyen, afirman e institucionalizan (22). Los hombres experimentan un nivel más alto de estrés relacionado con el empleo, pero es menos probable que busquen soportes externos (23). Este estudio realizó un análisis para evaluar la relación entre el autorreporte de bienestar mental o emocional del hombre con distinto factores, demostrando que la satisfacción con la vida sexual, percepción de la situación económica, la calidad del sueño y el apoyo social se relacionan con la variable resultado. Existe evidencia que indica riesgo asociado a la masculinidad, lo que sugiere que, a menudo, los hombres preferirán enfrentar el riesgo y la incomodidad física en vez de verse asociado con los rasgos percibidos como femenino, como la vulnerabilidad, la dependencia y la debilidad (34).

Una fortaleza del estudio corresponde a que los datos de la encuesta ENCAVI
2015-2016 provienen de un diseño muestral que permite la representatividad a nivel nacional; por lo tanto, los resultados obtenidos en este estudio podrían extrapolarse a la población de hombres en Chile. Para la obtención de los resultados que se presentan en este estudio, se utilizaron preguntas de diferentes secciones de la encuesta. Como se mencionó, las variables de resultado corresponden a dos preguntas de la encuesta, las que se utilizan como proxy para el bienestar emocional. Esto es una limitación que debe tenerse en cuenta a la hora de interpretar los resultados, ya que una evaluación más acabada y precisa del bienestar emocional de los hombres y de los factores que lo afectan requiere la utilización de un instrumento creado para dicho fin.

Por último, es necesario avanzar en la investigación de la salud de los hombres, proporcionar orientación para iniciativas de políticas, educación, prestación de servicios de salud y promoción de la salud dirigidas específicamente hacia ellos.

Conflicto de intereses Ninguno declarado por las autoras.

Declaración Las opiniones expresadas en este manuscrito son responsabilidad del autor y no reflejan necesariamente los criterios ni la política de la RPSP/ PAJPH y/o de la OPS.

\section{REFERENCIAS}

1. Courtenay WH. Constructions of masculinity and their influence on men's wellbeing: a theory of gender and health. Soc Sci Med. 2000;50(10):1385-401.

2. Services United State Department of Health and Human Services. Healthy people 2020. Washington; 2013.

3. Alfonso Urzúa M, Caqueo-Urízar A. Calidad de vida: una revisión teórica del concepto. Ter Psicol. 2012;30(1):61-71.

4. Diener E, Michalos AC, Glatzer W, Moum $\mathrm{T}$, Vogel J, Veenhoven R. Introduction Measuring well-being: collected theory and review works. En: Assessing WellBeing. 2009:1-6. Disponible en: http:// dx.doi.org/10.1007/978-90-481-2354-4

5. Magyar-Moe JL. Positive psychological interventions. En: Therapist's Guide to Positive Psychological Interventions. SAn Diego, CA: Elservier Academic Press; 2009:73-176

6. Obiols M, Núria S, Escoda P. Bienestar emocional, satisfacción en la vida y felicidad. En: Álvarez, M y Bisquerra, $\mathrm{R}$ (Coords). Manual de orientación $\mathrm{y}$ tutoría (versión electrónica, 3/2011). Barcelona: Wolters Kluwer Educación; 2011. Disponible en: http://www. $\mathrm{ub}$. ed u / g r o p / w p - content / uploads / 2014/03/Bienestar_emocional_satisfaccion_en_la_v.pdf Acceso el 18 de mayo de 2018.

7. Weich S, Brugha T, King M, McManus S, Bebbington P, Jenkins R, et al. Mental well-being and mental illness: findings from the adult psychiatric morbidity survey for England 2007. Br J Psychiatry. 2011;199(1):23-8.

8. Garfield CF, Isacco A, Rogers TE. A review of men's health and masculinity. AJLM. 2008;2:474-87.

9. Connell RW. Gender, men and masculinities. EOLSS. 2009:140-155.

10. Gomez EG. Equidad, género y salud: retos para la acción. Rev Panam Salud Publica. 2002;11(5):435-8. Disponible en: http://libaccess.mcmaster.ca/login?url=http:/ / search.ebscohost.com/login.aspx?direct $=$ true $\& \mathrm{db}=\operatorname{cin} 20 \& \mathrm{AN}=106982712 \&$ site $=\mathrm{e}-$ host-live \&scope $=$ site
11. Gritz ER, Thompson B, Emmons K, Ockene JK, McLerran DF, Nielsen IR. Gender differences among smokers and quitters in the working well trial. Prev Med (Baltim). 1998;27(4):553-61.

12. Courtenay WH. Behavioral factors associated with disease, injury, and death among men: evidence and implications for Prevention. J Mens Stud. 2000;9(1):81-142.

13. Grant BF, Dawson DA. Introduction to the National Epidemiologic Survey on Alcohol and Related Conditions. Alcohol Res Heal. 2006;29(2):74-8.

14. Jané-Llopis E, Matytsina I. Mental health and alcohol, drugs and tobacco: a review of the comorbidity between mental disorders and the use of alcohol, tobacco and illicit drugs. Drug Alcohol Rev. 2006; 25(6):515-36.

15. Good GE [Ed], Brooks GR [Ed]. The new handbook of psychotherapy and counseling with men: a comprehensive guide to settings, problems, and treatment approaches. San Francisco, CA: JosseyBass; 2005. 
16. César KG, Takada LT, Brucki SMD, Nitrini $R$, Nascimento LFC, Oliveira MO, et al. Prevalence of depressive symptoms among elderly in the city of Tremembé, Brazil: Preliminary findings of an epidemiological study. Dement Neuropsychol. 2013;7(3):252-7.

17. Cunha RV da, Bastos GAN, Duca GF Del. Prevalência de depressão e fatores associados em comunidade de baixa renda de Porto Alegre, Rio Grande do Sul. Rev Bras Epidemiol. 2012;15(2):346-54.

18. Borges LJ, Benedetti TRB, Xavier AJ, d'Orsi E. Fatores associados aos sintomas depressivos em idosos: estudo EpiFloripa. Rev Saude Publica. 2013;47(4):701-10.

19. Connell RW, Messerschmidt JW. Hegemonic masculinity: rethinking the concept. Gend Soc. 2005;19(6):829-59.

20. Windmöller N, Zanello V. Depression and masculinities: a systematic literature review of Brazilian journals. Psicol em Estud. 2016;21(3):437-49.

21. Helgeson VS. The effects of masculinity and social support on recovery from myocardial infarction. Psychosom Med. 1991;53(6):621-33.

22. Stergiou-Kita M, Mansfield E, Colantonio A, Moody J, Mantis S. What's gender got to do with it? Examining masculinities, health and safety and return to work in male dominated skilled trades. Work. 2016;54(3):721-33.

23. Williams DR. The health of men: structured inequalities and opportunities. American Journal of Public Health. 2003; 93:724-31.
24. Ministerio de Salud Chile. Encuesta de Calidad de Vida y Salud. ENCAVI 20152016. Santiago de Chile; 2017.

25. Saunders JB, Aasland OG, Babor TF, De La Fuente JR, Grant M. Development of the Alcohol Use Disorders Identification Test (AUDIT): WHO Collaborative Project on Early Detection of Persons with Harmful Alcohol Consumption-II. Addiction. 1993;88(6):791-804.

26. Ministerio de Economía de Chile. Ley $\mathrm{N}^{\circ} 17374$ 10-DIC-1970. Biblioteca del Congreso Nacional. Disponible en: https: / / www.leychile.cl/Navegar?idNorma $=28960$

27. Orm Saab KA, Camacaro Cuevas M Determinantes socioculturales que condicionan la masculinidad y su impacto en la salud sexual y reproductiva de los hombres. Comunidad y Salud. 2013;11:27-36. Disponible en: http:/ / www.scielo.org.ve/ scielo.php?script $=$ sci_arttext $\&$ pid $=$ S1690-32932013000100005

28. Pallahuala US, Avendaño AS, Estrada GC. De la tradición y la evolución: bienestar psicológico en hombres patagónicos contemporáneos. Magallania (Punta Arenas). 2011;39(1):71-81. Disponible en: http://www.scielo.cl/scielo.php?script$=$ sci_arttext\&pid $=$ S0718-2244201100 0100004\&lng $=$ en\&nrm $=$ iso\&tlng $=$ en

29. Aguayo F, Correa P, Cristi P. Resultados de la Encuesta Internacional de Masculinidades y Equidad de Género (IMAGES) de Chile. Santiago de Chile; 2011.

30. Courtenay WH. Key feterminants of the health and well-being of men and boys. Int J Mens Health. 2003;2(1):1-30.
31. Krumm S, Checchia C, Koesters M, Kilian $\mathrm{R}$, Becker T. Men's views on depression: a systematic review and metasynthesis of qualitative research. Psychopathology. 2017;50:107-24.

32. Centro de Estudios de Conflicto y Cohesión Social (COES). Resultados Primera Ola, Estudio Longitudinal Social de Chile (ELSOC). Módulo 6: Salud y bienestar. Salud Mental en el Chile de hoy. Notas COES de Política Pública N¹5. Santiago, Chile; 2018.

33. Appleton S, Gill T, Taylor A, McEvoy D, Shi Z, Hill C, et al. Influence of gender on associations of obstructive sleep apnea symptoms with chronic conditions and quality of life. Int J Environ Res Public Health. 2018;15(5):930. Disponible en: http://www.ncbi.nlm.nih.gov/pubmed/29735909 Acceso el 19 de mayo de 2018.

34. Courtenay WH. Engendering health: a social constructionist examination of men's health beliefs and behaviors. Psychol Men Masc. 2000;1(1):4-15. doi:10.1037/1524-9220. 1.1.4

Manuscrito recibido el 19 de noviembre de 2017 Aceptado para su publicación, tras revisión, el 2 de julio de 2018.
SUMMARY

Factors associated with self-reporting of mental and emotional well-being in men in Chile

Keywords
Objective. Determine factors associated with the mental and emotional well-being of men in Chile.

Methods. Cross-sectional analytic study in men aged 15 years and older in Chile. Data from the National Survey on Quality of Life and Health 2015-2016 (ENCAVI) were used. The variables of interest were: perception of emotional and mental wellbeing, and self-reporting of depression or anxiety. The prevalence of each of these variables was estimated and logistic regression was used to evaluate their association with sociodemographic factors, habits, and health.

Results. Mental well-being or emotional health was perceived as being "less than good" by $22.6 \%$ of men (CI95\%: 20.3\%-25.0\%), while $4.9 \%$ (CI95\%: $3.8 \%-6.4 \%$ ) reported medically diagnosed depression. Perceived poor emotional well-being was associated with reporting less than good sexual satisfaction, with an odds ratio (OR) of 4.42 (CI95\%: 3.40-5.76), followed by poor quality of sleep and less social support than desired. Similar results were found in men who self-reported depression or anxiety; in this group, the highest probability of these results was associated with less than good quality of sleep, the presence of at least one chronic disease, and low sexual satisfaction.

Conclusion. The principal factor associated with self-reporting of mental or emotional well-being was dissatisfaction with sexual activity. This result suggests that masculine identity is linked with sexual performance. It is necessary to continue investigating men's health and provide guidance for health policy initiatives.

Men; masculinity; men's health; Chile. 
RESUMO Objetivo. Determinar os fatores associados ao bem-estar mental ou emocional entre homens no Chile.

Métodos. Estudo analítico transversal em indivíduos do sexo masculino com idade

Fatores associados ao autorrelato de bem-estar mental ou emocional entre homens no Chile

Palavras-chave de 15 anos ou mais no Chile. Foram usados dados da Pesquisa Nacional de Qualidade de Vida e Saúde 2015-2016 (ENCAVI). As variáveis de interesse foram percepção do bem-estar emocional ou mental e autorrelato de depressão ou ansiedade. Foi estimada a prevalência de cada uma destas variáveis e, com o uso de regressão logística, foi examinada a associação das variáveis de interesse com fatores sociodemográficos e outros fatores relacionados a hábitos e saúde.

Resultados. Dos participantes, 22,6\% (intervalo de confiança de 95\% [IC95\%] 20,3\%$25,0 \%$ ) referiram bem-estar mental ou emocional "regular" e 4,9\% (IC95\% 3,8\%-6,4\%) informaram diagnóstico médico de depressão. A percepção de bem-estar emocional menor foi associada ao relato de satisfação sexual "regular", com odds ratio (OR) de 4,42 (IC95\% 3,40-5,76), e qualidade de sono e apoio social "abaixo do ideal". Resultados semelhantes foram observados entre os indivíduos que autorrelataram depressão ou ansiedade. Houve uma tendência maior deste autorrelato ser associado a qualidade de sono "regular", presença de uma ou mais doenças crônicas e menor satisfação sexual.

Conclusão. O principal fator associado ao autorrelato de bem-estar mental ou emocional foi insatisfação com a vida sexual. Este achado corrobora que a identidade masculina está vinculada ao desempenho sexual. É necessário aprofundar as pesquisas de saúde do homem e obter subsídios para direcionar as iniciativas de políticas de saúde.

Homens; saúde do homem; masculinidade; Chile. 